\title{
Large-conductance calcium-dependent potassium channels prevent dendritic excitability in neocortical pyramidal neurons
}

\author{
Narimane Benhassine • Thomas Berger
}

Received: 30 January 2008 /Revised: 25 June 2008 / Accepted: 29 July 2008 /Published online: 2 September 2008

(C) Springer-Verlag 2008

\begin{abstract}
Large-conductance calcium-dependent potassium channels (BK channels) are homogeneously distributed along the somatodendritic axis of layer 5 pyramidal neurons of the rat somatosensory cortex. The relevance of this conductance for dendritic calcium electrogenesis was studied in acute brain slices using somatodendritic patch clamp recordings and calcium imaging. BK channel activation reduces the occurrence of dendritic calcium spikes. This is reflected in an increased critical frequency of somatic spikes necessary to activate the distal initiation zone. Whilst BK channels repolarise the somatic spike, they dampen it only in the distal dendrite. Their activation reduces dendritic calcium influx via glutamate receptors. Furthermore, they prevent dendritic calcium electrogenesis and subsequent somatic burst discharges. However, the time window for coincident somatic action potential and dendritic input to elicit dendritic calcium events is not influenced by BK channels. Thus, BK channel activation in layer 5 pyramidal neurons affects cellular excitability
\end{abstract}

Electronic supplementary material The online version of this article (doi:10.1007/s00424-008-0569-3) contains supplementary material, which is available to authorized users.

$\mathrm{N}$. Benhassine $\cdot \mathrm{T}$. Berger

Institute of Physiology, University of Bern,

Bühlplatz 5,

CH-3012 Bern, Switzerland

\section{N. Benhassine $(\square)$}

Brain Research Institute, University of Zürich,

Winterthurerstrasse 190,

CH-8057 Zürich, Switzerland

e-mail: benhassine@hifo.uzh.ch

\section{T. Berger}

Institute of Physiology and Pathophysiology, University of Mainz,

Duesbergweg 6,

D-55128 Mainz, Germany primarily by establishing a high threshold at the distal action potential initiation zone.

Keywords BK channels · Neocortical pyramidal neurons . Dendrite $\cdot$ Critical frequency $\cdot$ Rat $\cdot$ Calcium-dependent potassium channels $\cdot$ Calcium action potential . Pyramidal neuron $\cdot$ Sensorimotor cortex

\section{Introduction}

The apical dendrite of layer 5 pyramidal neurons is an excitable structure, characterised by a high threshold action potential (AP) initiation zone in the distal tuft [43]. Neocortical layer 5 neurons receive qualitatively different inputs along their somatodendritic axis [1]. In the trigeminal system, tactile information is primarily relayed in the thalamus which projects via different pathways to the deep granular layers of the neocortex. These thalamic inputs are directly and indirectly projecting onto the soma and the basal dendrites of the layer 5 pyramidal neurons [8]. This sensory information can give rise to a sodium spike at the somatic AP initiation zone [28] which is not only propagated along the axon but also backpropagated into the apical dendrite [47]. Associative intracortical inputs impinge on the distal portion of the apical dendrite. Neither a backpropagating somatic AP alone nor isolated associative inputs to the distal tuft are usually sufficient to elicit a dendritic calcium AP. However, the coincidence of a backpropagating AP with an excitatory input to the distal dendrite effectively halves the threshold for a dendritic AP (BAC firing; [25]). Once a calcium AP is initiated in the distal dendrite, it propagates forward to the soma where it can induce in turn a short burst of sodium APs. The mutual activation of both AP initiation zones (somatodendritic interaction) switches the 
spiking pattern of the cell from regular spiking to burst discharge [27].

Dendritic conductances are decisive for the integrative properties of pyramidal cells in the hippocampus and neocortex. Dendritic A-type potassium and hyperpolarisationactivated conductances $[5,19,32]$ determine spike shape and passive membrane properties, respectively. Thus, these conductances have an impact on suprathreshold and subthreshold signals. Large-conductance calcium-activated potassium channels (BK channels; [31, 39, 41]) participate in the AP repolarisation $[12,46]$. In CA1 pyramidal cells, BK channels shape the somatic AP but do not influence its backpropagation [37]. In contrast, in the same cell type, Golding and coworkers [15] showed that BK channel activation curtails dendritic calcium events. Functional BK channels were found to be homogeneously distributed all along the somatodendritic axis of layer 5 pyramidal neurons [3]. The fast kinetics of BK channels (1 ms activation time constant and $3 \mathrm{~ms}$ deactivation time constant under $10 \mu \mathrm{M}$ free calcium), the high opening probability (0.69 under $10 \mu \mathrm{M}$ free calcium) and the absence of inactivation [3] suggest a possible opening of these channels in the time course of a single sodium AP. Furthermore, the voltage and calcium dependence of these channels suggest that the emergence of a calcium AP in the distal dendrite could massively open dendritic BK channels. Their strong calcium dependence suggests in addition that subthreshold depolarisations can activate $\mathrm{BK}$ channels only if this depolarisation is accompanied by a local but massive increase in intracellular calcium (half-maximal activation of the BK channels at -65 and $+48 \mathrm{mV}$ for 10 and $1 \mu \mathrm{M}$ calcium, respectively; [3]). This study presents evidence that BK channels activated by depolarisation and calcium influx of both distal dendritic inputs and backpropagating APs enhance the threshold for the initiation of the dendritic calcium spike without affecting the temporal window of its activation by coincident dendritic and somatic inputs. This effect is due to a hyperpolarisation of the distal dendrite after the activation of BK channels, whilst a reduced AP propagation seems to be of less importance. Thus, BK channel activation is in the position to reduce the dendritic excitability in layer 5 pyramidal neurons.

\section{Materials and methods}

Brain slice preparation and cell identification Three hundred-micrometer-thick parasagittal slices of the somatosensory cortex were prepared from 28- to 37-day-old Wistar rats. This was carried out according to national and institutional guidelines to minimise pain and discomfort of the animals. Rats were deeply anaesthetised with halothane and killed by decapitation. The brain was removed rapidly, chilled in $0-4{ }^{\circ} \mathrm{C}$ extracellular solution and then sliced using a vibratome (Dosaka Microslicer DTK-1000, Kyoto, Japan). Slices were incubated at $37^{\circ} \mathrm{C}$ for $15 \mathrm{~min}$, and then left at room temperature until recording. Layer 5 pyramidal neurons from the somatosensory area with a thick apical dendrite were visualised by infrared differential interference contrast video microscopy utilising a Newvicon camera (C2400, Hamamatsu, Hamamatsu City, Japan) and an infrared filter (RG9, Schott, Mainz, Germany) mounted on an upright microscope (Axioskop FS, Zeiss, Oberkochen, Germany).

Electrophysiology Current clamp recordings were made from the soma or simultaneously from the soma and the apical dendrite of rat neocortical layer 5 pyramidal neurons in the whole-cell configuration (Figs. 1, 2, 3 and 4). An Axoprobe 1A amplifier (Axon Instruments, Union City, CA, USA) or a BVC-700A amplifier (Dagan, Minneapolis, MN, USA) were used. Electrodes were made from borosilicate glass tubing (Hilgenberg, Malsfeld, Germany) using a micropipette puller (P-87; Sutter Instrument,

Fig. 1 BK channels reduce the likelihood of occurrence and the amplitude of dendritic calcium APs following somatic spike trains. a Four consecutive APs were induced in the soma with DC current injection (6 nA, $2 \mathrm{~ms}$ ). Their frequency was either insufficient (below the critical frequency; left traces) or sufficient (beyond the critical frequency; right traces) to elicit a dendritic calcium AP under control conditions (black traces). Data were simultaneously recorded at soma (top traces) and dendrite (lower traces; $550 \mu \mathrm{m}$ from the soma). In the somatic recording, the dendritic calcium AP is reflected as an afterdepolarisation. The blockade of BK channels with $1 \mu \mathrm{M}$ paxilline (gray traces) increases the amplitude (right traces) and decreases the threshold (left traces) of the dendritic calcium AP as seen in a decrease of the critical frequency. b Upper part relative reduction in the critical frequency obtained from the somatic afterdepolarisation, following BK channel blockade. Lower part the area under the curve of the dendritic signal from eight cells is plotted against the somatic AP frequency and normalised to the value obtained under control conditions at $143 \mathrm{~Hz}$. c Same as in a, following blockade of BK channels with $100 \mathrm{nM}$ iberiotoxin (gray traces). Dendritic recording at $370 \mu \mathrm{m}$ from the soma. $\mathbf{d}$ As in $\mathbf{a}$, a train of four spikes was induced in the soma followed by a single spike with a 1-s delay (upper traces). The spike train had frequencies of 83,111 and $166 \mathrm{~Hz}$. Single spikes and spike trains below a critical frequency induce only small changes in intracellular calcium concentration monitored with calcium imaging at $600 \mu \mathrm{m}$ from the soma (OGB-1, $200 \mu \mathrm{M}$ ). Beyond the critical frequency under control conditions (black traces), a large dendritic calcium event is evoked under control $(166 \mathrm{~Hz})$. One micromolar paxilline decreases this critical frequency to $111 \mathrm{~Hz}$ (gray traces). The calcium increase due to a spike train beyond the critical frequency becomes even larger under paxilline. e Left part the dendritic change in fluorescence due to the spike train was plotted against the spiking frequency. This plot reflects the larger increase in intracellular calcium concentration following a stimulation capable to activate the distal calcium AP initiation zone. Right part relative change in the critical frequency of somatic APs, recorded with calcium imaging at the distal apical dendrite (between 690 and $750 \mu \mathrm{m}$ from the soma) following BK channel blockade 
Novato, CA, USA). Their resistance was $10-16 \mathrm{M} \Omega$ for dendritic and 6-8 M $\Omega$ for somatic recordings when filled with the intracellular solution. The access resistance, measured after gigaseal formation and every $10 \mathrm{~min}$, was stable during the course of the experiment. The access resistance was $26.0 \pm 6.7$ and $34.7 \pm 5.0 \mathrm{M} \Omega$ for somatic and dendritic recordings, respectively, at the beginning of the experiment and $27.0 \pm 7.3$ and $34.7 \pm 4.7 \mathrm{M} \Omega$ for somatic and dendritic recordings, respectively, at the end of the experiment (mean recording time $=52 \pm 14 \mathrm{~min}, n=7$ somatodendritic recordings). Recordings were rejected if the access resistance increased by more than $10 \%$ of the initial value. Data were low-pass filtered at $3 \mathrm{kHz}$ using the internal filter of the amplifier. The sampling frequency was $10-20 \mathrm{kHz}$. Data were digitised and stored on-line using Clampex 9 (Axon Instruments). Data were analysed off-line with Clampfit9. Bridge balance and capacitance compensation was used in all recordings. Single APs were induced by
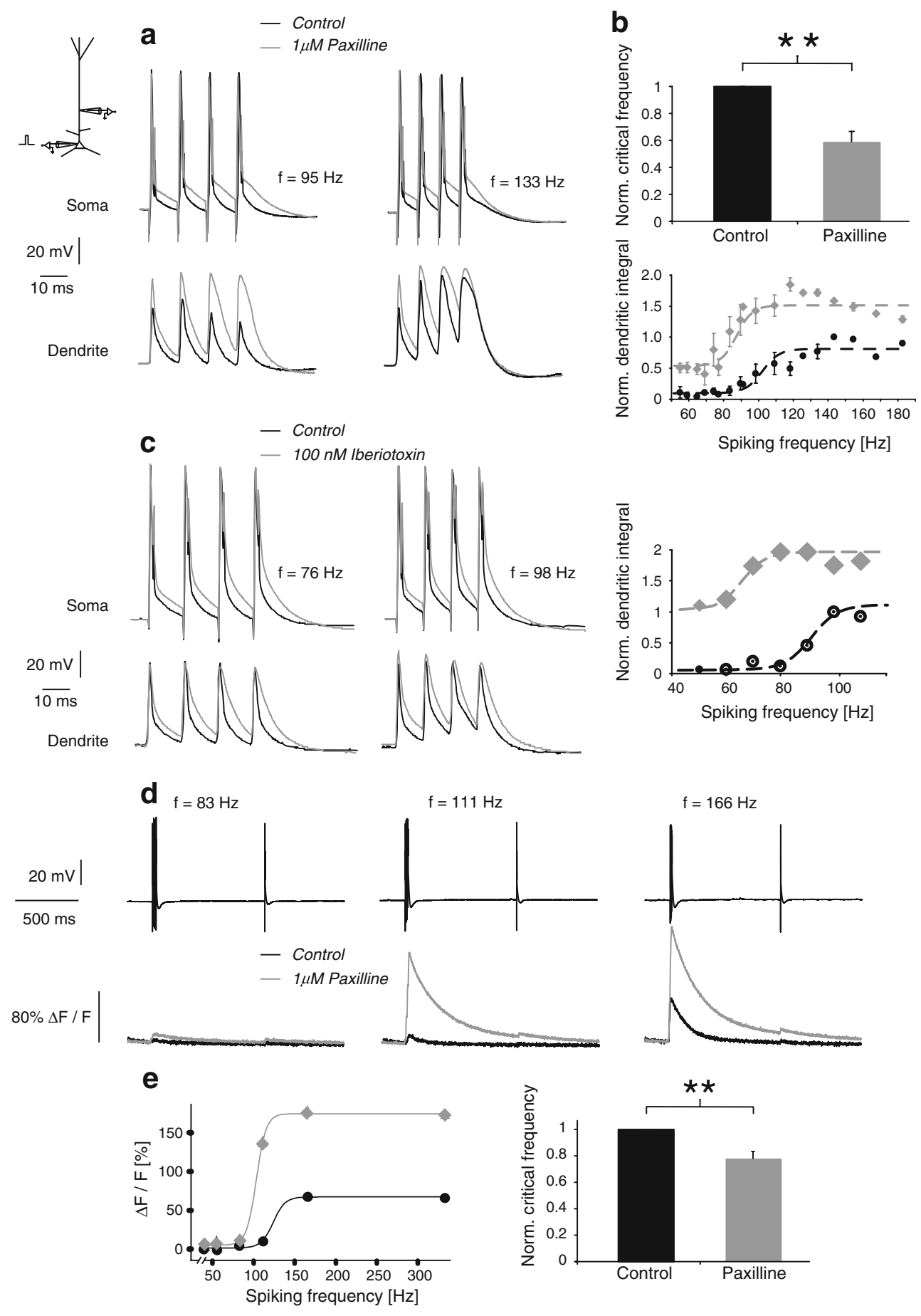
Fig. 2 BK channels participate in the somatic AP repolarisation and contribute weakly to its dendritic attenuation. a Single

APs were induced with a somatic DC current injection (5 nA, $4 \mathrm{~ms}$ ). Traces were aligned and stimulation artefacts removed. Relative increase in somatic AP width at half peak amplitude under control and with BK channel blockade $(n=6$ cells). b The change in somatic calcium concentration during an AP is increased following blockade of BK channels (OGB$1,200 \mu \mathrm{M})$. Relative change under paxilline ( $n=7$ cells).

c Top panel averaged APs evoked by DC current pulse at the soma (400 ms; $0.2-0.5 \mathrm{nA}$ ) and recorded simultaneously in the soma (left traces) and in the dendrite at different locations from the soma (right traces). The dendritic recordings were obtained from four cells at different dendritic recording distances indicated. Middle panel dendritic calcium transients evoked by a single backpropagated AP are larger after BK channel blockade (data taken from two cells imaged at different dendritic locations). Lower panel AP peak amplitude and half-width are normalised to the somatic AP and plotted as a function of the dendritic location under control and paxilline conditions. The blockade of BK channels reduces slightly the peak attenuation at distal dendritic locations, whilst the half-width is less affected a
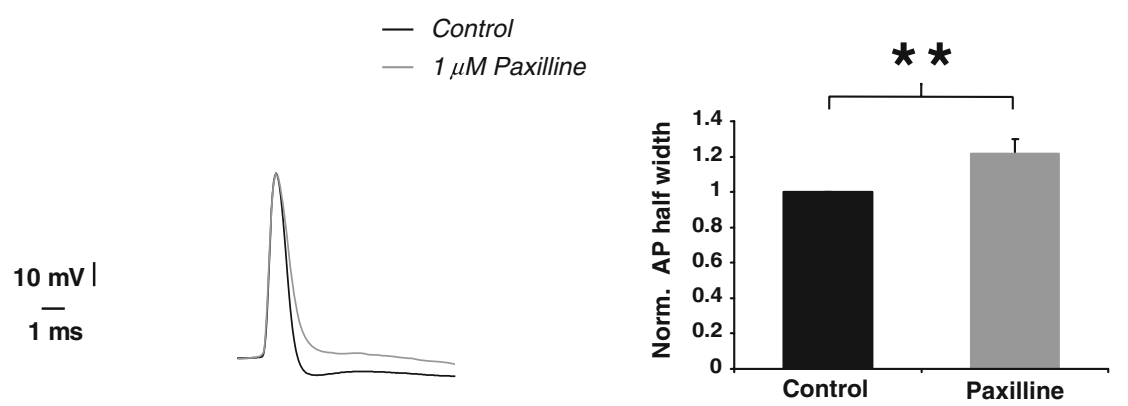

b

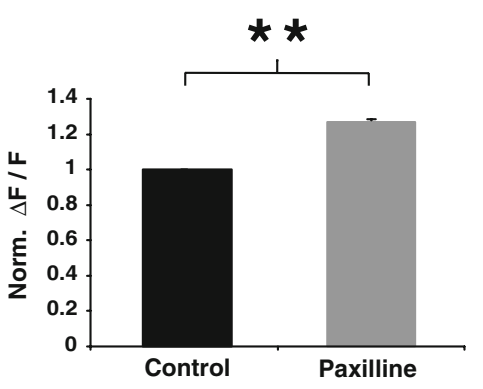

C
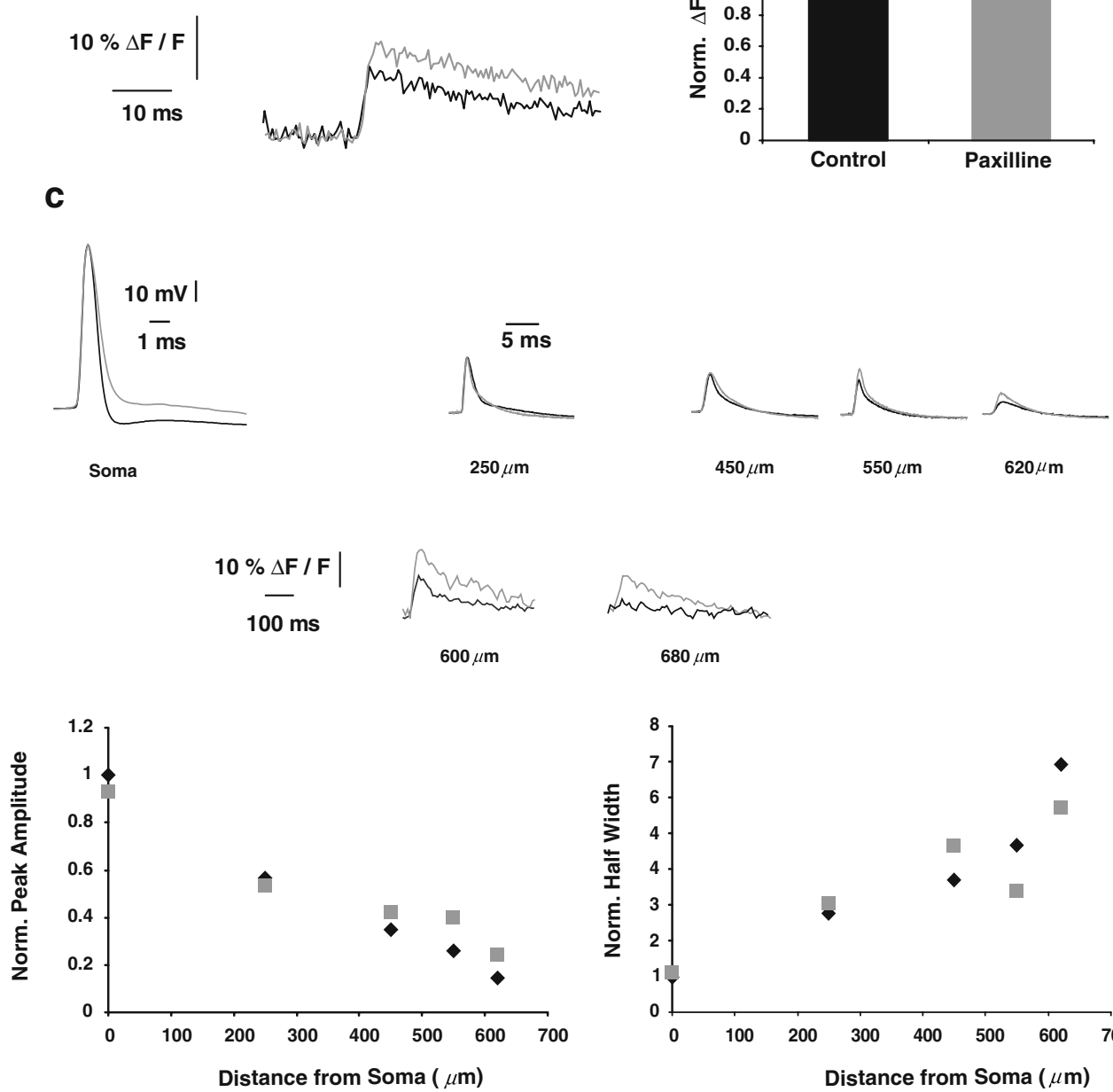

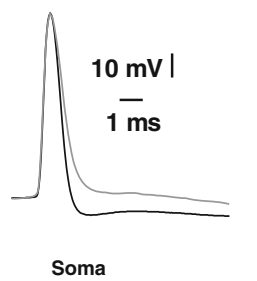

$250 \mu \mathrm{m}$

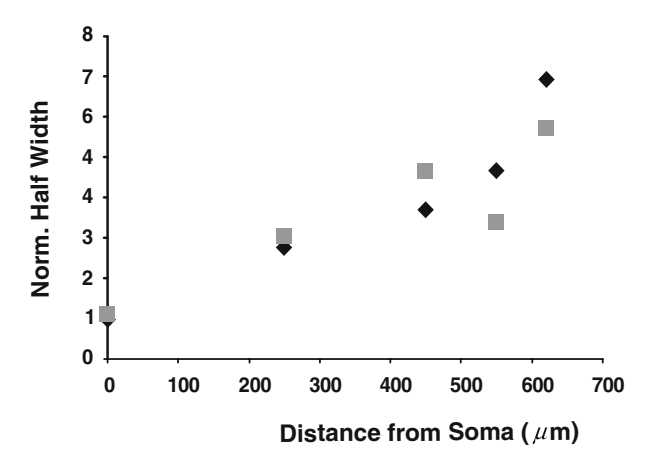

suprathreshold short DC current pulses (5-6 nA, 2-4 ms duration) injected into the soma (Fig. 2a). In order to subtract the depolarisation due to the passive membrane properties and stimulation artefacts, the cell was then hyperpolarised until the same current injection failed to elicit an action potential. Ten traces obtained either at the original or at the hyperpolarised membrane potential were averaged. Average traces obtained by DC pulse injection from a hyperpolarised potential were subtracted from the average traces obtained by
DC pulse injection from the resting membrane potential. Averaged action potentials under control conditions and BK channel blockade were aligned to the AP threshold defined by the initial rise of the AP derivative. The values for AP half-width under control conditions and BK channel blockade were normalised and compared for statistical significance using ANOVA for within-group comparison with significance levels set to 0.05 or 0.01 . The same statistical test was used for the analysis of the fluorescent 
Fig. 3 Postsynaptic BK channels reduce glutamate-induced calcium influx in the distal dendrite. a Fluorescence image of a layer 5 pyramidal neuron visualised using excitation of the calcium-insensitive dye Alexa 594. The calcium imaging location is marked by a white rectangle. The positions of the glutamate iontophoresis electrode and of the recording electrode are indicated. b Top panel averaged dendritic $\left[\mathrm{Ca}^{2+}\right]_{\mathrm{i}}$ signals (OGB-1, $200 \mu \mathrm{M}$ ) induced by dendritic glutamate iontophoresis (500 mM, $0.1 \mathrm{~ms}, 3 \mathrm{~V}$ pulse; top traces) and corresponding electrophysiological averaged trace recorded from the soma (lower traces) under control (black traces) and bath application of $1 \mu \mathrm{M}$ paxilline (gray traces). In few cases, the postsynaptic potential showed an increased inhibitory component after paxilline application (inset, arrowhead). Lower panel relative peak values of $\left[\mathrm{Ca}^{2+}\right]_{\mathrm{i}}$ signals induced by glutamate iontophoresis before and after $\mathrm{BK}$ channel blockade. Paxilline increases the glutamate-induced dendritic calcium influx ( $p=0.004 ; n=3$ cells)

peak induced by a somatic AP (Fig. 2b) and for the analysis of the minimal spiking frequency of a train of somatic APs capable to elicit a dendritic calcium AP (critical frequency; Fig. 1b). This same test was also used for the analysis of the fluorescent peak in the dendrite induced by a somatically triggered dendritic calcium AP (Fig. 1e) and for the analysis of the fluorescence peak induced by glutamate iontophoresis on the dendrite (Fig. 3). Dendritic excitability was studied by injection of $400 \mathrm{~ms}$ long DC pulses $(0.2-1.6 \mathrm{nA}$ in $0.1 \mathrm{nA}$ steps) in the dendrite (Fig. 4). The AP threshold was defined as the first current step able to induce a somatic AP. Pooled data are expressed as the mean \pm standard deviation (SD). All experiments were done at $34^{\circ} \mathrm{C}$.

Calcium imaging The hexapotassium salt of the calciumsensitive dye Oregon Green BAPTA-1 ( $K_{\mathrm{d}}$ value $170 \mathrm{nM}$; Molecular Probes, Eugene OR, USA) was used to monitor changes in cytosolic calcium concentration, whilst membrane voltage changes were recorded simultaneously. Two hundred micromolar of Oregon Green BAPTA was added to the patch pipette solution and diffused freely into the cell interior. This concentration allowed the filling of the dendritic structures of a layer 5 pyramidal cell within 30$45 \mathrm{~min}$. Changes in intracellular calcium were imaged with a Zeiss Axioskop 2FS+ microscope equipped with $\times 40 / 0.8$ NA optics. The light source was a 75-W Xenon short arc lamp in a monochromator (Deltaram V, PTI, Lawrenceville NJ, USA) equipped with a shutter. Excitation of the dye was obtained at $480 / 15 \mathrm{~nm}$. A dichroic mirror $(510 \mathrm{~nm})$ and an emission filter BP540/25 nm were used to detect the emitted light. The increase in fluorescence of the nonratiometric dye Oregon Green BAPTA reflecting an increase in the intracellular calcium concentration could be well-detected without any binning or averaging with a low-resolution, fast CCD camera (Redshirt NeuroCCD, RedShirt Imaging, Fairfield CT, USA). The CCD camera was running under Neuroplex with an un-binned full frame rate of $1 \mathrm{kHz}$ and a field of view of $280 \times 280 \mu \mathrm{m}$ (un- a

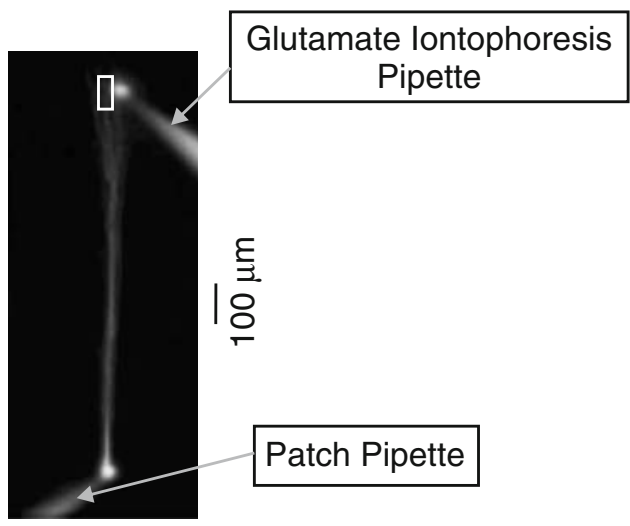

b
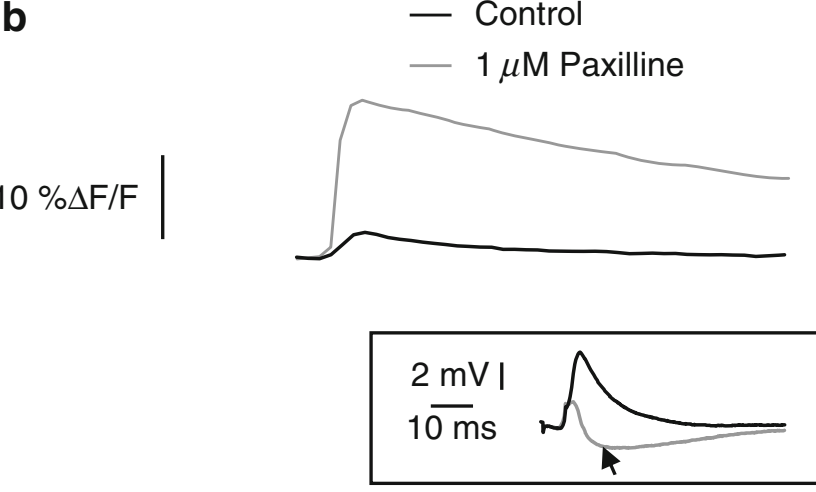

$1 \mathrm{mV}$

$20 \mathrm{~ms}$

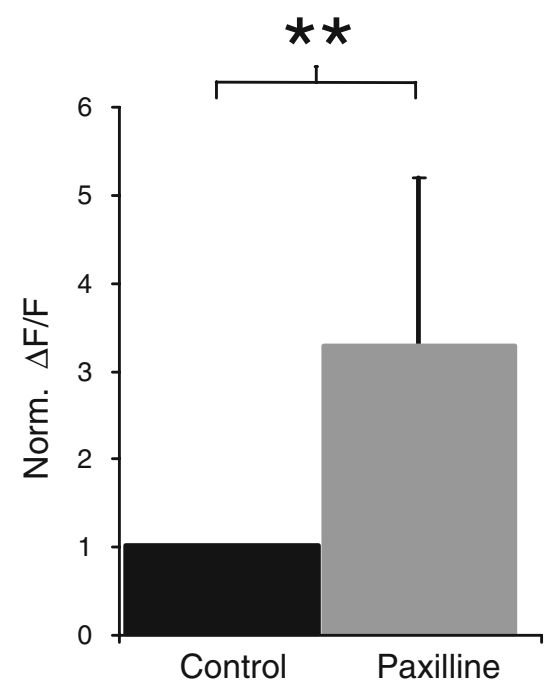

binned pixel field $3.5 \times 3.5 \mu \mathrm{m}, 80 \times 80$ pixels). Imaging data were digitised and stored on a PC using the Neuroplex software (Figs. 1d, 2b,c, 3 and 5).

Glutamate iontophoresis In iontophoresis experiments, the cytosol of layer 5 pyramidal cells was filled with Oregon 
Fig. 4 BK channels impair dendritically evoked burst discharges. a DC current pulses were injected in the dendrites of two pyramidal cells $(400 \mathrm{~ms}$ duration; 640 and $400 \mu \mathrm{m}$ from the soma). Recordings from the soma (right traces) and from the dendrite (left traces) were obtained under control (black traces) or with $1 \mu \mathrm{M}$ paxilline (gray traces). b Same as in a, but BK channels were blocked by $100 \mathrm{nM}$ iberiotoxin (dendritic recording at $370 \mu \mathrm{m}$ from the soma). BK channel blockade enables the induction of dendritic calcium events resulting in somatic bursts discharges
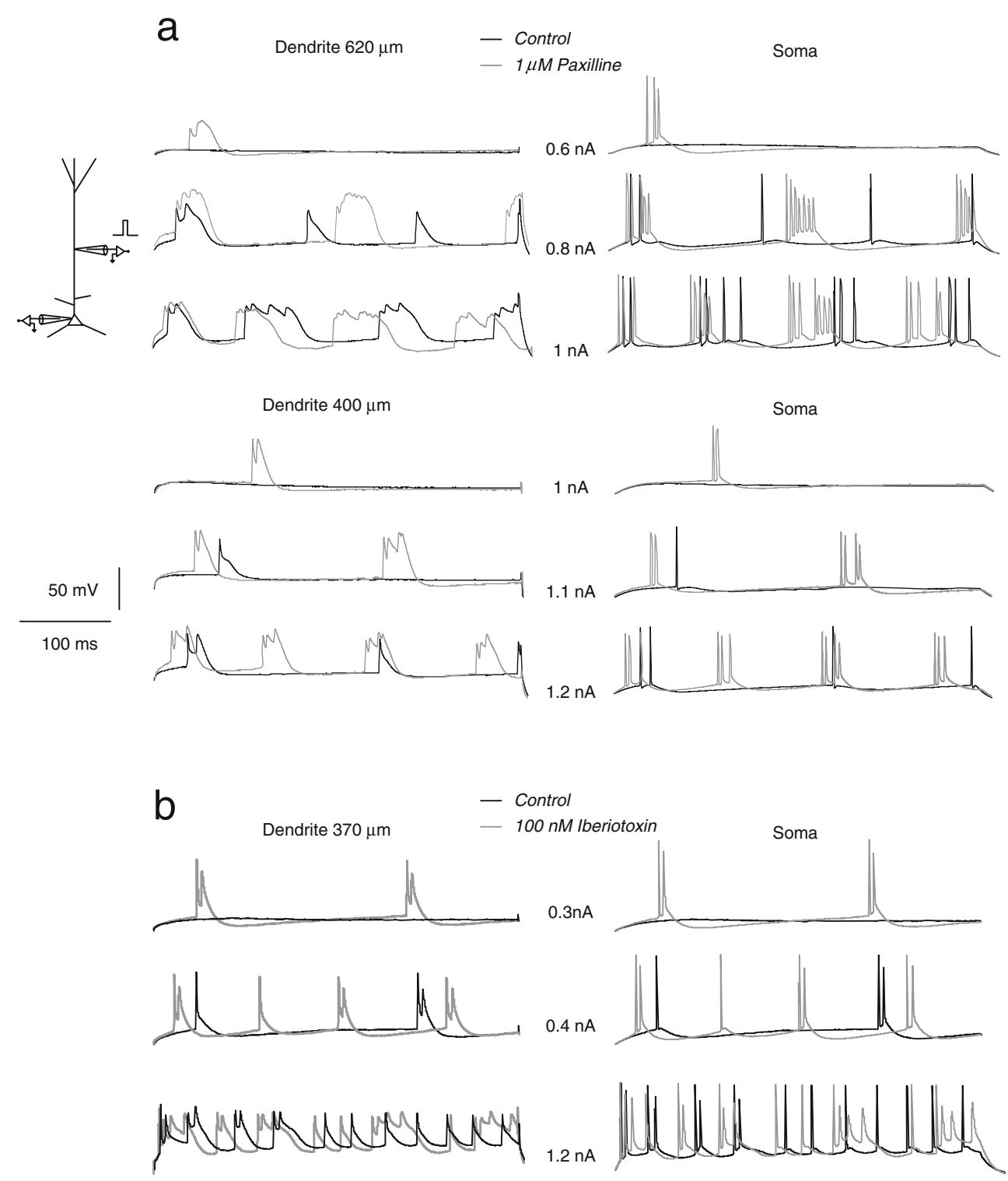

Green BAPTA-1 and the calcium-insensitive dye Alexa 594 (Molecular Probes; excitation $560 \mathrm{~nm}$, dichroic mirror $580 \mathrm{~nm}$, emission filter LP590 nm). A iontophoretic glass pipette with the tip size of a somatic patch pipette was advanced onto the distal dendritic arbor of the recorded layer 5 pyramidal neuron at a distance of 700-750 $\mu \mathrm{m}$ from the soma. Five hundred millimolar glutamate was applied iontophoretically with $0.1 \mathrm{~ms}$ long pulses ranging from +2 to $+20 \mathrm{~V}$ (Figs. 3 and 5).

Chemicals and solutions Slices were continuously superfused with a physiological extracellular solution containing (in millimolar): $125 \mathrm{NaCl}, 25 \mathrm{NaHCO}_{3}, 2.5 \mathrm{KCl}, 1.25$ $\mathrm{NaH}_{2} \mathrm{PO}_{4}, 2 \mathrm{CaCl}_{2}, 1 \mathrm{MgCl}_{2}, 20$ glucose, 5 pyruvate, bubbled with $95 \% \mathrm{O}_{2}$ and $5 \% \mathrm{CO}_{2}$. For current clamp experiments without additional calcium imaging (Figs. 1a- c, 2a, 4, Supplementary Figs. 1a and 2), the pipette solution contained (in millimolar): $110 \mathrm{~K}$-gluconate, $30 \mathrm{KCl}, 10$ EGTA, 10 HEPES, $5 \mathrm{CaCl}_{2}, 4 \mathrm{Mg}$-ATP, $0.3 \mathrm{Na}_{2}$-GTP, $10 \mathrm{Na}_{2}$-phosphocreatine (free calcium concentration of $100 \mathrm{nM}$ ). Pipette solution for combined current clamp recording and calcium imaging (Figs. 1d, 2b,c, 3 and 5) contained: $135 \mathrm{~K}$-gluconate, $5 \mathrm{KCl}, 4 \mathrm{Mg}$-ATP, $0.3 \mathrm{Na}_{2}$ GTP, $10 \mathrm{Na}_{2}$-phosphocreatine, 10 HEPES, 0.2 Oregon Green BAPTA-1. For iontophoretic application experiments (Figs. 3 and 5), the patch pipette solution contained in addition the calcium-insensitive dye Alexa Fluor $594(15 \mu \mathrm{M}$; Molecular Probes). The iontophoresis pipette contained $500 \mathrm{mM}$ glutamate and $15 \mu \mathrm{M}$ Alexa Fluor 594 diluted in extracellular solution. The $\mathrm{pH}$ was adjusted in the extracellular and the intracellular solutions to 7.3. Osmolarity of the intracellular solutions was in the range of 315 to 320 mOsm. 


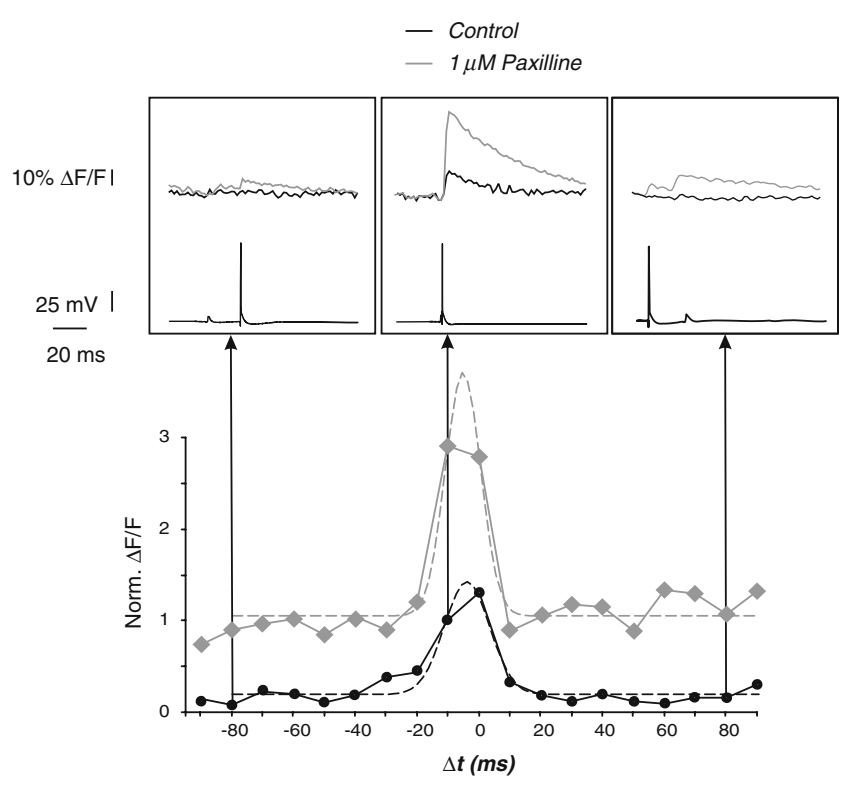

Fig. 5 BK channels reduce the amplitude but not the time window for induction of the calcium spike triggered by coincident somatic and dendritic inputs. Upper panel change in intradendritic calcium concentration (dendritic calcium imaging at $670 \mu \mathrm{m}$ from the soma, top traces in insets) and electrophysiological traces (lower traces in insets) induced by a combination of a single backpropagating AP (DC current pulse $1 \mathrm{nA}, 1 \mathrm{~ms}$ ) and a dendritic EPSP induced by glutamate iontophoresis on the distal dendrite $(700 \mu \mathrm{m}$ from the soma) under control (black traces) and paxilline (gray traces) conditions. Lower panel all $\Delta F / F$ values for both conditions are normalised to that obtained under control conditions with a $\Delta t$ of $-10 \mathrm{~ms} . \Delta t$ represents the time between the onset of the somatic current injection triggering the backpropagated AP and the onset of the glutamate iontophoresis stimulation. Changes in fluorescence are fitted by Gaussian functions (dashed lines) of different peak amplitude but similar mean and sigma (mean $-3.8 \mathrm{~ms}$ and sigma $7.4 \mathrm{~ms}$ under control; mean $-5.1 \mathrm{~ms}$ and sigma $4.7 \mathrm{~ms}$ under paxilline for the cell shown here)

The high affinity and selective BK channel blockers paxilline [22] and iberiotoxin [14] were purchased from Alomone Labs (Jerusalem, Israel). All other drugs were from Sigma or Merck. Paxilline and iberiotoxin were first dissolved in dimethyl sulphoxide (DMSO) at $1 \mathrm{mM}$ and bidistillated water at $100 \mu \mathrm{M}$, respectively. Aliquots of these stock solution were kept at $-20^{\circ} \mathrm{C}$ and diluted 1:1,000 in the external solution directly before use. Paxilline or iberiotoxin at final concentrations of $1 \mu \mathrm{M}$ and $100 \mathrm{nM}$, respectively, were bath applied with the extracellular solution. In the paxilline experiments, the final concentration of DMSO was $0.1 \%$, a concentration that was found to have no effect on BK channels in control experiments. The effective BK channel blockade with paxilline occurred between 2 and 4 min after the change of the perfusion (Supplementary Fig. 3). A similar time course was observed for the perfusion of extracellular solution containing iberiotoxin. Data obtained in the first $10 \mathrm{~min}$ following change of perfusate were not used for evaluation.

\section{Results}

BK channels limit dendritic excitability following somatic action potentials

A dendritic calcium AP can be initiated by coincident dendritic and somatic input (BAC firing mechanism; [25]) or alternatively by a train of backpropagating somatic APs at a frequency higher than a critical value (critical frequency, CF; [24]). To study the impact of BK channel activation on dendritic excitability, whole-cell current clamp experiments were performed using a potassiumbased intracellular solution containing a free calcium concentration of $100 \mathrm{nM}$ mimicking physiological calcium levels [18]. We induced dendritic calcium APs with trains of four somatic spikes triggered by somatic current injections $(6 \mathrm{nA}, 2 \mathrm{~ms}$, frequency range from 49 to $400 \mathrm{~Hz}$ ). This was done in control conditions and after BK channel blockade with the specific blockers paxilline or iberiotoxin (Fig. 1a,c) [14, 22]. In the somatic recording, the dendritic calcium AP is reflected as a prominent afterdepolarisation (Fig. 1a; compare to [24]). The dendritic calcium AP appears at a significantly lower CF under paxilline $(1 \mu \mathrm{M})$ in comparison to control conditions. In somatodendritic patch clamp recordings (mean distance $540 \mu \mathrm{m}$ from the soma), paxilline reduces the $\mathrm{CF}$ to $56.6 \%$ of the control $(127.1 \pm 35.7 \mathrm{~Hz}$ under control conditions and $71.9 \pm 13.9 \mathrm{~Hz}$ under paxilline; $p=0.003 ; n=7$ cells; Fig. 1a, b). In addition, paxilline increases the amplitude and integral of the calcium AP recorded in the dendrite (Fig. 1a,b). Similarly, the blockade of BK channels with $100 \mathrm{nM}$ iberiotoxin lowers the $\mathrm{CF}$ to $76.4 \pm 1.6 \%$ of the control $(n=3$ cells $)$ and increases the integral of the dendritic calcium AP (Fig. 1c). Application of $1 \mu \mathrm{M}$ paxilline in addition to $100 \mathrm{nM}$ iberiotoxin does not intensify the effects observed under iberiotoxin alone.

In additional experiments, we have combined somatic current clamp recordings with calcium imaging experiments in the soma and apical dendrite. Changes in the intracellular calcium concentration were induced by trains of four somatic action potentials below and above the $\mathrm{CF}$ as well as single spikes (Fig. 1d). The somatic calcium concentration increases with the number of spikes and the spiking frequency (control values; single spike $=11.5 \pm 7.9 \% \Delta F / F$; spike trains below the $\mathrm{CF}=26.4 \pm 14.5 \% \Delta F / F$; spike trains above the $\mathrm{CF}=35.7 \pm 18.1 \% \Delta F / F ; n=7$ cells, not shown). The calcium transients at the calcium AP initiation zone $(580 \pm 172 \mu \mathrm{m}$ distal to the soma; compare to [26]) are small for single backpropagating spikes (mean $6.1 \% \Delta F / F$, range between $0.6 \% \Delta F / F$ at $900 \mu \mathrm{m}$ and $12.24 \% \Delta F / F$ at $400 \mu \mathrm{m} ; n=5$ imaging positions in three cells) and for trains of backpropagating spikes below the CF (mean $11.3 \% \Delta F$ / $F$, range between $0.8 \% \Delta F / F$ at $900 \mu \mathrm{m}$ and $22.1 \% \Delta F / F$ 
at $400 \mu \mathrm{m} ; n=5$ imaging positions in three cells). However, calcium transients due to spike trains above the $\mathrm{CF}$ are large (mean $61.0 \% \Delta F / F$, range between $22.6 \% \Delta F / F$ and $102.2 \% \Delta F / F ; n=5$ imaging positions in three cells) (Fig. 1d). The small calcium transients are due to single backpropagating spikes or groups of backpropagating spikes with a low frequency insufficient to activate the dendritic calcium AP zone, whilst the large transients are due to an activation of the calcium AP zone. In this set of experiments, under control condition, a frequency of $116.6 \pm$ $23.1 \mathrm{~Hz}$ is found to be critical for the initiation of calcium spikes (Fig. 1d,e; $n=8$ cells). Blockade of BK channels with $1 \mu \mathrm{M}$ paxilline resulted in a large increase of the intracellular calcium for spike trains above the CF (151.2 \pm $4.3 \%$ of the control at somatic and $202.7 \pm 37.4 \%$ of the control at dendritic imaging sites; $n=2$ somatic and $n=5$ dendritic imaging positions; $p=0.017$ for all seven recordings; Fig. 1d,e). The $\mathrm{CF}$ in the distal apical dendrite is reduced to $77.6 \%$ of the control $(116.6 \pm 23.1 \mathrm{~Hz}$ under control conditions and $90.6 \pm 8.4 \mathrm{~Hz}$ under paxilline; $p=$ $0.007 ; n=8$ cells; Fig. 1e). Also, under iberiotoxin application, the amplitude of the dendritic calcium event following somatic spikes above the CF increases to $188.1 \pm$ $63.6 \%$ of the control and reduced the CF to $76.4 \%$ of the control ( $n=3$ cells; not shown). These data show a clear role of BK channels in setting a high threshold for dendritic calcium action potentials induced by somatic activity.

BK channels shorten the somatic action potential and tend to reduce its backpropagation in layer 5 pyramidal cells

Different mechanisms may underlie the reduced impact of somatic spike trains on dendritic calcium AP generation following BK channel activation. One possibility is the modulation of the somatic AP shape. A sodium spike of a reduced half-width undergoes stronger attenuation during its backpropagation (compare to [6]) and is less likely to elicit a calcium spike in the distal dendrite. In order to test this, we induced somatic APs under control conditions and with BK channel blockade. Figure 2a shows averaged AP waveforms from the same cell following stimulus artefact and leak subtraction under both conditions. The spike under $1 \mu \mathrm{M}$ paxilline shows a significant prolongation (halfwidth $=0.71 \pm 0.03 \mathrm{~ms}$ under control, $0.87 \pm 0.05 \mathrm{~ms}$ with paxilline; $p=0.004 ; n=6$ cells). This is mainly due to a reduction of the fast afterhyperpolarisation of the AP underlying the repolarisation slope. In addition, paxilline application increases the somatic intracellular calcium concentration accompanying isolated APs (Fig. 2b). These data demonstrate the physiological activation of $\mathrm{BK}$ channels during a single somatic AP, an effect already expected due to the fast activation kinetics found on the single channel level [3].
In a next step, we have investigated whether BK channel activation could affect the ability of the somatic spike to backpropagate into the apical dendrite. We used simultaneous whole-cell recordings on the soma and the apical dendrite of layer 5 pyramidal neurons and compared the dendritic changes in AP peak amplitude and half-width due to BK channel blockade (Fig. 2c). Backpropagating APs were evoked by DC pulse current injection at the soma (400 ms; 0.2 to $0.5 \mathrm{nA}$, four dendritic recording locations between 250 and $620 \mu \mathrm{m}$ ). The backpropagating AP undergoes a distance-dependent peak attenuation and increase in half-width. In the distal dendrite $(620 \mu \mathrm{m}$ from soma), the AP peak amplitude was $13 \mathrm{mV}$ under control and $21 \mathrm{mV}$ under BK blockade. This depolarisation should be insufficient to open high-voltage calcium channels and, therefore, should fail to activate dendritic BK channels. However, in the distal dendrite only $(>400 \mu \mathrm{m})$, the blockade of BK channels tends to reduce the peak amplitude attenuation. Changes in the half-width of the backpropagating AP in the distal portion of the apical dendrite are less clear (Fig. 2c). In another set of experiments, the calcium transients induced by the backpropagation of single APs or trains of APs below the CF were studied in the distal dendrite. Calcium transients due to single spikes increase to $182.1 \pm 88.0 \%$ of the control after BK channel blockade (Fig. 2c middle panel). Similarly, the calcium transients for spike trains below the $\mathrm{CF}$ increase to $175.8 \pm 61.7 \%$ of the control $(n=5$ dendritic recordings in three cells; distance 400 to $900 \mu \mathrm{m}$ from the soma; not shown). The dendritic calcium transients have comparable half-widths under control and in paxilline. But one has to have in mind that the kinetics of imaged calcium transients are primarily a function of the unbinding of calcium from the dye and not of the underlying changes in membrane potential or calcium concentration [16]. In addition, results obtained with calcium imaging are always hampered by the fact that calcium-sensitive dyes do not respond in a linear fashion to the changes in membrane potential [4]. Taken together, these results show that BK channels tend to reduce the AP backpropagation in the distal apical dendrite.

BK channels reduce dendritic calcium influx via ligand-gated conductances

In addition to backpropagating APs in the apical dendrite, BK channels may also be activated by dendritic inputs opening glutamate-gated NMDA receptor channels. BK channels would prevent the depolarisation necessary to activate dendritic calcium channels and thereby the dendritic calcium action potential discharge. We mimicked an excitatory input on the distal apical dendrite of layer 5 pyramidal neurons $(690-750 \mu \mathrm{m}$ from the soma; Fig. 3a). Following iontophoretic glutamate application $(500 \mathrm{mM}$; 
0.1 ms duration; +2 to $+20 \mathrm{~V}$ pulse, $n=3$ cells; compare to [44]), we imaged the changes in dendritic calcium concentration (Fig. 3b). Glutamate iontophoresis induces a rise in intradendritic calcium concentration which is significantly enhanced following an overall blockade of BK channels $(\triangle F / F=1.9 \pm 0.9 \%$ under control, $3.7 \pm 2.4 \%$ under paxilline, $p=0.004$; Fig. $3 \mathrm{~b}$ and insets of Fig. 5). Due to the amplitude of these evoked calcium transients in comparison to those obtained with a dendritic calcium AP (range between $22.6 \% \Delta F / F$ and $102.2 \% \Delta F / F$ under control conditions; Fig. 1d), it seems probable that this procedure evokes only a local calcium transient but is insufficient to evoke directly a dendritic calcium AP. Whilst subthreshold activity without increase in intracellular calcium is insufficient to activate dendritic BK channels (Supplementary Fig. 1), the local depolarisation due to distal glutamatergic inputs and the concomitant rise in intradendritic calcium due to the NMDA-type glutamate receptor activation seem to be sufficient to activate dendritic BK channels. In four out of 11 applications, the postsynaptic potentials evoked by the distal glutamate ejection and recorded at the soma had smaller amplitude after paxilline application than under control conditions, suggesting an increased inhibitory component (Fig. 3b, inset, arrowhead). The global blockade of BK channels by bath application of paxilline may have changed the inhibitory postsynaptic component evoked by glutamate iontophoresis. Even though the blockade of BK channels in neocortical GABAergic interneurons does not affect their discharge properties [11], BK channels have been predominantly localised in axons and presynaptic terminals, including those of GABAergic interneurons [33]. Thus, the BK channel blockade might have facilitated the neurotransmitter release in this cell type. However, even in these cases, the BK channel blockade led to a bigger increase in distal dendritic fluorescence. In addition, the latency between the glutamate iontophoresis stimulation and the onset of the somatically recorded subsequent excitatory postsynaptic potential (EPSP) was identical for all cells between control and BK blockade conditions, suggesting that the increased change in intracellular calcium concentration could not be due to a variation of the iontophoresis pipette position after paxilline treatment. Dendritic BK channels dampen the depolarisation following distal glutamatergic inputs, preventing the activation of calcium conductances and, therefore, setting a high threshold for the distal action potential initiation zone.

BK channels prevent dendritically evoked burst discharges

DC current pulses $(400 \mathrm{~ms}, 0.2-1.6 \mathrm{nA})$ were injected in the apical dendrite of layer 5 pyramidal neurons (450$620 \mu \mathrm{m}$ from the soma) and the voltage responses were simultaneously recorded at both somatic and dendritic locations (Fig. 4). Following BK channel blockade, a dendritic current injection is more likely to induce somatic spikes compared to control conditions (somatic spike threshold reduced to $82.5 \pm 0.13 \%$ of the control; $n=5$ cells). Moreover, in four out of six cells, this dendritic current injection leads to tonic somatic spike discharges under control, but induces doublets or high-frequency burst discharges due to a BAC firing mechanism when BK channels are blocked by paxilline or iberiotoxin (Fig. 4). We saw no evidence of a favoured propagation of the dendritic depolarisation to the soma following BK channel blockade. Indeed, if EPSP-like waveforms (peak amplitude $1,000 \mathrm{pA}$, time to peak $2.5 \mathrm{~ms}$, decay time constant $5.7 \mathrm{~ms}$; Supplementary Fig. 1a, upper trace) are injected in the dendrite $(300 \mu \mathrm{m}$ from the soma), the blockade of BK channels does not affect the EPSP propagation to the soma (Supplementary Fig. 1a, middle trace). In addition, this result cannot be explained by a modification of the somatic discharge threshold if the BK conductance is blocked. Indeed, neither the somatic nor the dendritic input resistance was changed after BK channel blockade (somatic input resistance $=26.2 \pm 8.0 \mathrm{M} \Omega$ under control and $27.1 \pm$ 8.7 $\mathrm{M} \Omega$ under paxilline; $p=0.88 ; n=5$ cells; dendritic input resistance $=19.4 \pm 4.7 \mathrm{M} \Omega$ under control and $19.2 \pm 4.0 \mathrm{M} \Omega$ under paxilline; $p=0.74 ; n=6$ cells). In addition, $1 \mu \mathrm{M}$ paxilline does not affect the mean spiking frequency of the cells following somatic DC current (400-1,200 pA, $18 \mathrm{~s}$ ) or Gaussian-distributed noisy current injection (mean $=800$ 1,200 pA; sigma $=200$ pA, 18 s) (Supplementary Fig. 2; $n=$ 4 cells). No change was found in the instantaneous frequency or in the spiking frequency adaptation under paxilline.

In the presence of paxilline, the same dendritic current injection changes the spiking pattern of some cells from a regular spiking discharge to a burst discharge (Fig. 4). BK channel blockade prevents the deactivation of calcium conductances allowing a lower threshold at the dendritic AP initiation zone. This result suggests a functional importance of BK channels in limiting the switch from a low level of excitability to a burst discharge pattern in layer 5 pyramidal neurons.

BK channels do not change the time window for BAC firing

The critical frequency paradigm used in Fig. 1 elicits a calcium spike in the distal dendrite using high-frequency somatic spikes discharges [24]. Such burst discharges of three to five APs at frequencies between 200 and $400 \mathrm{~Hz}$ have been reported in layer 5 pyramidal neurons in vivo firing spontaneously or following sensory stimulation [17]. The temporal coincidence of a backpropagating somatic AP 
and a distal dendritic depolarisation within an optimal time window (the distal depolarisation arriving 3 to $7 \mathrm{~ms}$ after the backpropagating AP) is the most probable way to induce a calcium AP (BAC firing; [25]). We studied this mechanism in layer 5 pyramidal neurons with somatic patch clamp recordings and simultaneous calcium imaging of the distal dendrite $(600-680 \mu \mathrm{m}$ from the soma). A combination of a backpropagating AP triggered by somatic current injection (DC pulse, $0.6-1 \mathrm{nA}, 1 \mathrm{~ms}$ ) and glutamate iontophoresis at the dendrite $(500 \mathrm{mM} ; 0.1 \mathrm{~ms} ;+2$ to $+20 \mathrm{~V}$ pulse) mimics a distal excitatory input. Using this, we have tested whether BK channels could affect the coincidence detection mechanism in layer 5 pyramidal neurons (Fig. 5).

As expected, the peak amplitude of calcium concentration rise in the dendrite is much bigger after BK channel blockade compared to control conditions (for coincident timing between the dendritic input and the somatic AP, the dendritic $\Delta F / F$ was $6.3 \pm 1.1 \%$ under control and $14.2 \pm$ $4.3 \%$ under paxilline conditions; $n=3$ cells). This pronounced increase in the distal dendritic calcium influx reinforces the idea of an activation of local BK channels by glutamate-gated conductances, preventing the opening of voltage-gated calcium conductances. In order to compare the time window for calcium AP induction under control and $\mathrm{BK}$ channel blockade conditions, the profile of the intradendritic change in calcium concentration was fitted with Gaussian functions (Fig. 5, lower part, dashed lines). Similar parameters can be used to fit the change in dendritic calcium concentration under both conditions (mean $-4.2 \mathrm{~ms}$ and sigma $10.1 \mathrm{~ms}$ under control; mean $-5.1 \mathrm{~ms}$ and sigma $8.2 \mathrm{~ms}$ under paxilline; $n=3$ cells). This result suggests that BK channels do not affect the time window for the induction of calcium APs in the case of coincident somatic and dendritic inputs. Despite a slight attenuation of AP propagation in the distal dendrite (Fig. 2), BK channel activation has no influence on the time window for the emergence of calcium spikes. Evidence shows BK channels in the distal dendrite act likely primarily locally to set a high threshold for the dendritic AP initiation zone. The temporal window of calcium AP activation by coincident dendritic and somatic inputs observed is consistent with previous reports [24]. The averaged latency between the onset of the glutamate iontophoresis stimulation and the rise of the corresponding EPSP observed at the soma was $8.5 \pm 0.3 \mathrm{~ms}$ ( $n=14$ in three cells). Taking into account an approximate conduction velocity of $0.5 \mathrm{~m} \mathrm{~s}^{-1}$ in the apical dendrite of layer 5 pyramidal neurons [27, 48], the EPSP is expected to peak in the distal dendrite about $7.1 \mathrm{~ms}$ after the onset of the iontophoresis stimulation. Using these corrections, we could observe, in accordance with previous reports [24], a maximal calcium rise in the distal dendrite occurring for a latency of $2.9 \mathrm{~ms}$ between the backpropagating AP and the distal EPSP under control conditions. We observe a real boost of the calcium transient amplitude when BK channels are blocked primarily due to a local dendritic effect on ligand- and voltage-gated calcium conductances. Thus, BK channels act in layer 5 pyramidal cells as effective filters under high levels of cellular activity. They dampen the dendritic excitability and prevent bursting in this cell type.

\section{Discussion}

BK channel activation in layer 5 pyramidal cells of the somatosensory cortex depends on two biophysical parameters, namely, depolarisation and increased intracellular calcium [3]. These properties limit the influence of this conductance to suprathreshold events like somatic sodium spikes and dendritic calcium action potentials if the calcium increase is moderate (about $1 \mu \mathrm{M}$ ). The specific activation pattern of BK channels, in combination with their even expression along the somatodendritic axis, shortens APs at the soma and reduces their ability to initiate dendritic action potentials during a rise in cellular excitability. If the calcium increase is localised and strong enough (about $10 \mu \mathrm{M}$ ), moderate depolarisations around the threshold are also sufficient for the activation of BK channels [3].

In layer 5 pyramidal neurons, as in other cell types, BK channels participate in spike repolarisation $[12,23,46$, this study]. Like BK channels [3], high-voltage-activated calcium channels are expressed at the soma and dendrite of layer 5 pyramidal neurons $[21,50]$. Furthermore, a colocalisation [7] and co-activation of BK channels and Ntype calcium channels has been reported in hippocampal and neocortical pyramidal neurons [29, 49]. As Sun et al. [49] have shown in mouse neocortical neurons, the opening of high-voltage-activated calcium channels during the rising phase of the AP provides a sufficient calcium influx for the activation of neighbouring BK channels (see also [34]). BK channel activation leads to the closing of voltagedependent depolarising sodium and calcium conductances and, therefore, hastens repolarisation. This acceleration of the AP decay was shown not to interfere with repetitive spiking of the cell because BK channels remain open only for a few milliseconds [3, 45].

In hippocampal pyramidal neurons, Poolos and Johnston [37] report an effect of BK channels on the AP repolarisation which is restricted to the somatic compartment and conclude that the distribution of BK channels is not uniform along the somatodendritic axis. In contrast, immuno-localisation of BK channels showed their presence in both postsynaptic and presynaptic compartments of hippocampal pyramidal neurons, i.e. also in dendritic spines [40]. BK channels in neocortical pyramidal cells show a uniform distribution along the somatodendritic axis. In 
addition, their biophysical properties such as their voltage and calcium dependence or their kinetics are similar in both compartments [3]. This suggests that BK channel activation during somatic and dendritic suprathreshold activity is not only able to shorten sodium but also calcium action potentials. In hippocampal CA1 pyramidal cells, dendritic calcium AP duration and initiation is controlled by $\mathrm{BK}$ channels among other potassium conductances [15]. In this study, we show that, in neocortical pyramidal neurons, BK channel activation prevents the generation and reduces the amplitude and duration of dendritic calcium APs.

Which are the mechanisms underlying the effect of BK channels on dendritic excitability? A faster repolarisation of the somatic AP reduces the AP backpropagation into the dendrite and, therefore, the excitability of the dendritic calcium AP initiation zone due to somatic activity (compare to [6]). In the distal dendrite, the AP filtering tends to be stronger under control conditions than under BK channel blockade. Furthermore, the calcium transients corresponding to the backpropagated spikes are larger following BK channel blockade. These findings suggest that BK channel activation tends to reduce the AP backpropagation, but this effect is of minor importance in regard of calcium spike generation.

Upon activation, the primary effect of BK channels is to change the membrane potential and thereby to affect the currents underlying the calcium AP. Indeed, BK channel activation reduced the overall integral of calcium APs. This finding is comparable to Purkinje cells where dendritic excitability is enhanced and the spread of dendritic calcium spikes is improved by the block of dendritic BK channels [9, 38, 51]. Following BK channel activation, we have shown a strong reduction of distal calcium influx triggered by glutamate application. Excitatory associative inputs arriving at distal positions on the apical dendrite of layer 5 pyramidal neurons activate dendritic glutamate receptors of the AMPA- and NMDA-type. In the olfactory bulb, NMDA receptors and BK channels are linked and their coactivation leads to membrane potential hyperpolarisation [20]. The calcium influx and the membrane depolarisation resulting from NMDA receptor activation during a dendritic EPSP presumably activate BK channels. This activation can lead consecutively to a closure of the NMDA channels via an increased magnesium block. In contrast, no activation of BK channels is found during dendritic EPSP-like waveforms. In this case, the depolarisation is not accompanied by a calcium influx. This lack of a calcium increase at the vicinity of the dendritic BK channels leads to the failure of BK channel activation. Another possible mechanism for the glutamatergic activation of BK channels is the activation of metabotropic glutamate receptors (mGluR). Indeed, the mGluR1 subtype is expressed on cell bodies and dendrites of neocortical neurons [30]. In cerebellar neurons, these receptors are found to activate BK channels [10]. Furthermore, other studies report an inhibition of the calcium signaling in hippocampal or neocortical pyramidal neurons following the activation of mGluR1 [13, 42]. This subtype of glutamatergic receptors may, therefore, modulate the excitability of the calcium AP initiation zone through mechanisms involving BK conductances.

In layer 5 pyramidal neurons, other voltage- and ligandgated conductances influence the emergence of calcium APs or BAC firing. Bekkers and Delaney [2] have shown that D-type potassium channels are expressed near the site of AP initiation. This strategic site of expression allows this conductance to regulate the somatic excitability and consequently the excitability of the dendritic AP initiation zone as well. Hyperpolarisation-activated channels have a higher density in the distal apical dendrite than the proximal region [5]. This $I_{\mathrm{h}}$ conductance limits the backpropagation of sodium APs as well as the summation of coincident dendritic inputs [35]. In this way, $I_{\mathrm{h}}$ increases the electrotonic distance between both AP initiation zone and impairs the induction of $\mathrm{BAC}$ firing in neocortical pyramidal neurons [6]. In contrast to BK channels, these leak conductances act at the resting membrane potential and can influence thereby not only APs but also the cellular integration at the subthreshold level. A ligand-gated conductance, which shunts dendritic activity, acts via $\mathrm{GABA}_{\mathrm{A}}$ and $\mathrm{GABA}_{\mathrm{B}}$ receptors. Concomitant activation of distal dendritic GABAergic inputs leads to the so-called veto effect preventing the generation of calcium APs during a long time window $[25,36]$. In this respect, BK channels and GABA receptors act comparably because both conductances directly impair the generation of the dendritic AP via a hyperpolarisation of the membrane potential.

The functional role of dendritic BK channels is of particular interest in layer 5 pyramidal neurons in the somatosensory cortex. These cells receive qualitatively different inputs along their somatodendritic axis. Tactile information is relayed via the thalamus, and excitatory spiny stellate cells in layer 4 , to the soma and the proximal dendrites of layer 5 pyramidal neurons. This sensory input can give rise to a sodium spike at the somatic AP initiation zone, which is backpropagated into the dendrite. On the other hand, inputs from associative cortical areas project on the distal apical dendrite of these neurons [1]. It is likely that neither backpropagated sodium APs nor associative inputs on their own induce a sufficient depolarisation to reach the high threshold of the dendritic AP zone ([27], but see $[17,26]$ for the generation of calcium APs by distal inputs alone in vivo). However, the coincidence of a backpropagated AP, together with an input on the distal dendrite, is the most likely condition to pass the dendritic threshold. This will induce a calcium AP and switch the output pattern of the cell from a regular spiking pattern to a 
burst discharge pattern. The triggering of calcium APs in these cells corresponds to a mechanism detecting the temporal coincidence of sensory and associative inputs. BK channels participate in setting a high threshold for the dendritic AP initiation zone by a direct curtailing of sodium and calcium APs. The activation of BK channels leads to an active decoupling of the somatic and the dendritic AP initiation zones during high-frequency input at somatic and/ or dendritic locations. BK channels would, therefore, modulate the switch between a low-excitability and a high-excitability mode and act as an active dampening filter defining the significance of coincident sensory and associative inputs.

Acknowledgements The results were extensively discussed with Drs. F. Helmchen, M. Giugliano, M. Larkum, H.R. Lüscher and D. Ulrich who, in addition, have read earlier versions of this manuscript. Supported by the Swiss National Science Foundation (grant 3100107529/1), as well as the Novartis Foundation for Medical-Biological Research and the Bonizzi-Theler Foundation to T.B.

\section{References}

1. Abeles M (1984) Corticonics - neural circuits of the cerebral cortex. Cambridge University Press, Cambridge

2. Bekkers JM, Delaney AJ (2001) Modulation of excitability by adendrotoxin-sensitive potassium channels in neocortical pyramidal neurons. J Neurosci 21:6553-6560

3. Benhassine N, Berger T (2005) Homogeneous distribution of large-conductance calcium-dependent potassium channels on soma and apical dendrite of rat neocortical layer 5 pyramidal neurons. Eur J Neurosci 21:914-926

4. Berger T, Borgdorff A, Crochet S, Neubauer FB, Lefort S, Fauvet B, Ferezou I, Carleton A, Lüscher HR, Petersen CC (2007) Combined voltage and calcium epifluorescence imaging in vitro and in vivo reveals subthreshold and suprathreshold dynamics of mouse barrel cortex. J Neurophysiol 97:3751-3762

5. Berger T, Larkum ME, Lüscher HR (2001) High $\mathrm{I}_{\mathrm{h}}$ channel density in the distal apical dendrite of layer 5 pyramidal cells increases bidirectional attenuation of EPSPs. J Neurophysiol 85: 855-868

6. Berger T, Senn W, Lüscher HR (2003) Hyperpolarizationactivated current $I_{h}$ disconnects somatic and dendritic spike initiation zones in layer $\mathrm{V}$ pyramidal neurons. J Neurophysiol 4:2428-2437

7. Berkefeld H, Sailer CA, Bildl W, Rohde V, Thumfart JO, Eble S, Klugbauer N, Reisinger E, Bischofberger J, Oliver D, Knaus HG, Schulte U, Fakler B (2006) $\mathrm{BK}_{\mathrm{Ca}}-\mathrm{Cav}$ channel complexes mediate rapid and localized $\mathrm{Ca}^{2+}$-activated $\mathrm{K}^{+}$signaling. Science 5799: $615-620$

8. Bureau I, von Saint Paul F, Svoboda K (2006) Interdigitated paralemniscal and lemniscal pathways in the mouse barrel cortex. PLoS Biol 4:2361-2371

9. Cavelier P, Pouille F, Desplantez T, Beekenkamp H, Bossu JL (2002) Control of the propagation of dendritic low-threshold $\mathrm{Ca}^{2+}$ spikes in Purkinje cells from rat cerebellar slice cultures. J Physiol (Lond) 540:57-72

10. Chavis P, Ango F, Michel JM, Bockaert J, Fagni L (1998) Modulation of big $\mathrm{K}^{+}$channel activity by ryanodine receptors and L-type $\mathrm{Ca}^{2+}$ channels in neurons. Eur J Neurosci 10:2322-2327
11. Erisir A, Lau D, Rudy B, Leonard CS (1999) Function of specific $\mathrm{K}+$ channels in sustained high-frequency firing of fast-spiking neocortical interneurons. J Neurophysiol 82:2476-2489

12. Faber ES, Sah P (2002) Physiological role of calcium-activated potassium currents in the rat lateral amygdala. J Neurosci 22: $1618-1628$

13. Frenguelli BG, Potier B, Slater NT, Alford S, Collingridge GL (1993) Metabotropic glutamate receptors and calcium signalling in dendrites of hippocampal CA1 neurones. Neuropharmacology 32:1229-1237

14. Galvez A, Gimenez-Gallego G, Reuben JP, Roy-Contancin L, Feigenbaum P, Kaczorowski GJ, Garcia ML (1990) Purification and characterization of a unique, potent, peptidyl probe for the high conductance calcium-activated potassium channel from venom of the scorpion Buthus tamulus. J Biol Chem 265:11083-11090

15. Golding NL, Jung HY, Mickus T, Spruston N (1999) Dendritic calcium spike initiation and repolarization are controlled by distinct potassium channel subtypes in CA1 pyramidal neurons. J Neurosci 19:8789-8798

16. Helmchen F, Imoto K, Sakmann B (1996) $\mathrm{Ca}^{2+}$ buffering and action potential-evoked $\mathrm{Ca}^{2+}$ signaling in dendrites of pyramidal neurons. Biophys J 70:1069-1081

17. Helmchen F, Svoboda K, Denk W, Tank DW (1999) In vivo dendritic calcium dynamics in deep-layer cortical pyramidal neurons. Nat Neurosci 11:989-996

18. Hille B (2001) Ionic channels of excitable membranes. Sinauer, Sunderland, MA

19. Hoffman DA, Magee JC, Colbert CM, Johnston D (1997) Potassium channel regulation of signal propagation in dendrites of hippocampal pyramidal neurons. Nature 387:869-875

20. Isaacson JS, Murphy GJ (2001) Glutamate-mediated extrasynaptic inhibition: direct coupling of NMDA receptors to $\mathrm{Ca}^{2+}$-activated $\mathrm{K}^{+}$channels. Neuron 6:877-879

21. Kelly KM, Kume A, Albin RL, Macdonald RL (2001) Autoradiography of L-type and $\mathrm{N}$-type calcium channels in aged rat hippocampus, entorhinal cortex, and neocortex. Neurobiol Aging 22:17-23

22. Knaus HG, McManus OB, Lee SH, Schmalhofer WA, GarciaCalvo M, Helms LM, Sanchez M, Giangiacomo K, Reuben JP, Smith AB 3rd (1994) Tremorgenic indole alkaloids potently inhibit smooth muscle high-conductance calcium-activated potassium channels. Biochemistry 33:5819-5828

23. Lancaster B, Nicoll RA (1987) Properties of two calcium-activated hyperpolarizations in rat hippocampal neurons. J Physiol (Lond) 389:187-203

24. Larkum ME, Kaiser KM, Sakmann B (1999a) Calcium electrogenesis in distal apical dendrites of layer 5 pyramidal cells at a critical frequency of back-propagating action potentials. Proc Natl Acad Sci USA 25:14600-14604

25. Larkum ME, Zhu JJ, Sakmann B (1999b) A new mechanism for coupling inputs arriving at different cortical layers. Nature 398: $338-341$

26. Larkum ME, Zhu JJ (2002) Signaling of layer 1 and whiskerevoked $\mathrm{Ca}^{2+}$ and $\mathrm{Na}^{+}$action potentials in distal and terminal dendrites of rat neocortical pyramidal neurons in vitro and in vivo. J Neurosci 22:6991-7005

27. Larkum ME, Zhu JJ, Sakmann B (2001) Dendritic mechanisms underlying the coupling of the dendritic with the axonal action potential initiation zone of adult rat layer 5 pyramidal neurons. $\mathrm{J}$ Physiol (Lond) 533:447-466

28. Manns ID, Sakmann B, Brecht M (2004) Sub- and suprathreshold receptive field properties of pyramidal neurones in layers $5 \mathrm{~A}$ and $5 \mathrm{~B}$ of rat somatosensory barrel cortex. J Physiol (Lond) 15:601-622

29. Marrion NV, Tavalin $\mathrm{J}$ (1998) Selective activation of $\mathrm{Ca}^{2+}$ activated $\mathrm{K}^{+}$channels by co-localized $\mathrm{Ca}^{2+}$ channels in hippocampal neurons. Nature 395:900-905 
30. Martin LJK, Blackstone CD, Huganir RL, Price DL (1992) Cellular localization of a metabotropic glutamate receptor in brain. Neuron 9:259-270

31. Marty A (1981) Calcium-dependent potassium channels with large unitary conductance in chromaffin cell membrane. Nature 291:497-500

32. Migliore M, Shepherd GM (2002) Emerging rules for the distributions of active dendritic conductances. Nat Rev Neurosci 3:362-370

33. Misonou H, Menegola M, Buchwalder L, Park EW, Meredith A, Rhodes KJ, Aldrich RW, Trimmer JS (2006) Immunolocalization of the $\mathrm{Ca}^{2+}$-activated $\mathrm{K}^{+}$channel Slo1 in axons and nerve terminals of mammalian brain and cultured neurons. J Comp Neurol 496:289-302

34. Müller A, Kukley M, Uebachs M, Beck H, Dietrich D (2007) Nanodomains of single $\mathrm{Ca}^{2+}$ channels contribute to action potential repolarization in cortical neurons. J Neurosci 27:483-495

35. Nicoll A, Larkman A, Blakemore C (1993) Modulation of EPSP shape and efficacy by intrinsic membrane conductances in rat neocortical pyramidal neurons in vitro. J Physiol (Lond) 468:693710

36. Perez-Garci E, Gassmann M, Bettler B, Larkum ME (2006) The GABAB1b isoform mediates long-lasting inhibition of dendritic $\mathrm{Ca}^{2+}$ spikes in layer 5 somatosensory pyramidal neurons. Neuron 50:603-616

37. Poolos NP, Johnston D (1999) Calcium-activated potassium conductances contribute to action potential repolarization at the soma but not the dendrites of hippocampal CA1 pyramidal neurons. J Neurosci 19:5205-5212

38. Rancz EA, Häusser M (2006) Dendritic calcium spikes are tunable triggers of cannabinoid release and short-term synaptic plasticity in cerebellar Purkinje neurons. J Neurosci 26:5428-5437

39. Sah P, Davies P (2000) Calcium-activated potassium currents in mammalian neurons. Clin Exp Pharmacol Physiol 27:657-663

40. Sailer CA, Kaufmann WA, Kogler M, Chen L, Sausbier U, Ottersen OP, Ruth P, Shipston MJ, naus HG (2006) Immuno- localization of BK channels in hippocampal pyramidal neurons. Eur J Neurosci 24:442-454

41. Sausbier U, Sausbier M, Sailer CA, Arntz C, Knaus HG, Neuhuber W, Ruth P (2006) $\mathrm{Ca}^{2+}$-activated $\mathrm{K}^{+}$channels of the BK-type in the mouse brain. Histochem Cell Biol 125:725-741

42. Sayer RJ (1998) Group I metabotropic glutamate receptors mediate slow inhibition of calcium current in neocortical neurons. J Neurophysiol 80:1981-1988

43. Schiller J, Schiller Y, Stuart G, Sakmann B (1997) Calcium action potentials restricted to distal apical dendrites of rat neocortical pyramidal neurons. J Physiol (Lond) 505:605-616

44. Schwindt PC, Crill WE (1995) Amplification of synaptic current by persistent sodium conductance in apical dendrite of neocortical neurons. J Neurophysiol 74:2220-2224

45. Shao LR, Halvorsrud R, Borg-Graham L, Storm JF (1999) The role of BK-type $\mathrm{Ca}^{2+}$-dependent $\mathrm{K}^{+}$channels in spike broadening during repetitive firing in rat hippocampal pyramidal cells. J Physiol (Lond) 521:135-146

46. Storm JF (1987) Action potential repolarization and a fast afterhyperpolarization in rat hippocampal pyramidal cells. J Physiol (Lond) 385:733-759

47. Stuart GJ, Sakmann B (1994) Active propagation of somatic action potentials into neocortical pyramidal cell dendrites. Nature 367:69-72

48. Stuart G, Schiller J, Sakmann B (1997) Action potential initiation and propagation in rat neocortical pyramidal neurons. J Physiol (Lond) 505:617-632

49. Sun X, Gu XQ, Haddad GG (2003) Calcium influx via L- and N-type calcium channels activates a transient large-conductance $\mathrm{Ca}^{2+}$-activated $\mathrm{K}^{+}$current in mouse neocortical pyramidal neurons. J Neurosci 23:3639-3648

50. Westenbroek RE, Hell JW, Warner C, Dubel SJ, Snutch TP, Catterall WA (1992) Biochemical properties and subcellular distribution of an N-type calcium channel $\alpha 1$ subunit. Neuron 9:1099-1115

51. Womack MD, Khodakhah K (2004) Dendritic control of spontaneous bursting in cerebellar Purkinje cells. J Neurosci 24:3511-3521 